\title{
Whole-genome single-nucleotide polymorphism (SNP) marker discovery and association analysis with the eicosapentaenoic acid (EPA) and docosahexaenoic acid (DHA) content in Larimichthys crocea
}

Shijun Xiao ${ }^{1}$, Panpan Wang ${ }^{1}$, Linsong Dong ${ }^{1}$, Yaguang Zhang ${ }^{1}$, Zhaofang Han ${ }^{1}$, Qiurong Wang ${ }^{1}$, Zhiyong Wang ${ }^{\text {Corresp. } 1}$

${ }^{1}$ Fisheries College, Jimei University, Xiamen, Fujian, China

Corresponding Author: Zhiyong Wang

Email address: zywang78@qq.com

Whole-genome single-nucleotide polymorphism (SNP) markers are valuable genetic resources for the association and conservation studies. Genome-wide SNP development in many teleost species are still challenging because of the genome complexity and the cost of re-sequencing. Genotyping-By-Sequencing (GBS) provided an efficient reduced representative method to squeeze cost for SNP detection; however, most of recent GBS applications were reported on plant organisms. In this work, we used an EcoRI-Nlalll based GBS protocol to teleost large yellow croaker, an important commercial fish in China and East-Asia, and reported the first whole-genome SNP development for the species. 69,845 high quality SNP markers that evenly distributed along genome were detected in at least $80 \%$ of 500 individuals. Nearly $95 \%$ randomly selected genotypes were successfully validated by SequenomMassARRAYassay. The association studies with the muscle eicosapentaenoic acid (EPA) and docosahexaenoic acid (DHA) content discovered 39 significant SNP markers, contributing as high up to $\sim 63 \%$ genetic variance that explained by all markers. Functional genes that involved in fat digestion and absorption pathway were identified, such as APOB, CRAT and OSBPL10. Notably, PPT2 Gene, previously identified in the association study of the plasma n-3 and n-6 polyunsaturated fatty acid level in human, was re-discovered in large yellow croaker. Our study verified that EcoRI-Nlalll based GBS could produce quality SNP markers in a cost-efficient manner in teleost genome. The developed SNP markers and the EPA and DHA associated SNP loci provided invaluable resources for the population structure, conservation genetics and genomic selection of large yellow croaker and other fish organisms. 
1 Whole-genome single-nucleotide polymorphism (SNP) marker discovery and

2 association analysis with the eicosapentaenoic acid (EPA) and

3 docosahexaenoic acid (DHA) content in Larimichthys crocea

4

5 Shijun Xiao ${ }^{1}$, Panpan Wang ${ }^{1}$, Linsong Dong ${ }^{1}$,Yaguang Zhang ${ }^{1}$, Zhaofang Han ${ }^{1}$, Qiurong Wang ${ }^{1}$, and Zhiyong 6 Wang ${ }^{1, *}$

7

81 Key Laboratory of Helthy Mariculture for the East China Sea, Ministry of Agriculture, P.R. China; Fishery

9 College, Jimei University, Yindou Road, Xiamen 361021, P.R. China

10

$11 *$ Corresponding author

12 Email: Zhiyong Wang - zywang@jmu.edu.cn;

13

14

15

16

17

18

19

20

21

22

23

24

25

26 


\section{Abstract}

Whole-genome single-nucleotide polymorphism (SNP) markers are valuable genetic resources for the association and conservation studies. Genome-wide SNP development in many teleost species are still challenging because of the genome complexity and the cost of re-sequencing. Genotyping-By-Sequencing (GBS) provided an efficient reduced representative method to squeeze cost for SNP detection; however, most of recent GBS applications were reported on plant organisms. In this work, we used an EcoRI-NlaIII based

34 GBS protocol to teleost large yellow croaker, an important commercial fish in China and East-Asia, and reported the first whole-genome SNP development for the species. 69,845 high quality SNP markers that evenly distributed along genome were detected in at least $80 \%$ of 500 individuals. Nearly $95 \%$ randomly selected genotypes were successfully validated by Sequenom MassARRAY assay. The association studies with the muscle eicosapentaenoic acid (EPA) and docosahexaenoic acid (DHA) content discovered 39 significant SNP markers, contributing as high up to $\sim 63 \%$ genetic variance that explained by all markers. Functional genes that involved in fat digestion and absorption pathway were identified, such as $A P O B, C R A T$ and OSBPL10. Notably, PPT2 Gene, previously identified in the association study of the plasma n-3 and n-6 polyunsaturated fatty acid level in human, was re-discovered in large yellow croaker. Our study verified that EcoRI-NlaIII based GBS could produce quality SNP markers in a cost-efficient manner in teleost genome. The developed SNP markers and the EPA and DHA associated SNP loci provided invaluable resources for the population structure, conservation genetics and genomic selection of large yellow croaker and other fish organisms. 


\section{Introduction}

Whole-genome single nucleotide polymorphism (SNP) is one of the most important genomic resources for population diversity, conservation genetics and functional gene identification for biological traits (Seeb et al. 2011). To obtain the molecular markers of the shared genomic loci among individuals, many technologies were invented and developed to probe whole-genome polymorphisms. The techniques allowing synthesizing DNA probes in chips have led to the advent and application of SNP microarrays (Lipshutz et al. 1999), making it possible to explore genome-wide SNP in a high-throughput manner. However the cost of array design and application obstructs the wider usage in non-model species, especially for endangered and economic organisms (De Donato et al. 2013). More importantly, microarray approaches cannot discover novel SNP loci for species without reference sequences (Popova et al. 2013). With the development of next-generation sequencing (NGS), the state-of-art sequencing platform enable scientists to scan small variants in genomes at an unprecedentedly scale with rapidly decreasing price. The multiplex library strategies were widely used to further reduce the cost per sample. However, the budget is still one of the biggest challenges for whole-genome re-sequencing in non-model samples (Muir et al. 2016). Furthermore, the whole-genome sequencing data for hundreds of individuals also inevitably burdens the limited computational and bioinformatics capacity in labs.

In the past few years, several robust sequencing-based genotyping techniques have been invented in the research community to overcome the bottle-neck of cost in whole-genome resequencing. Most of those innovations employ a strategy of partial genome representation sequencing (Narum et al. 2013), such as restriction site associated DNA (RAD) (Rowe et al. 2011), IIB restriction endonucleases based RAD (2bRAD) (Wang et al. 2012) and Genotyping-By-Sequencing (GBS) (De Donato et al. 2013). RAD applies a restriction enzyme to digest genome DNA and then random fragment them to generate RAD tags. Although RAD experiments was initially designed for microarray-based genotyping (Miller et al. 2007), the updated RAD tag isolation and library construction procedure has been prevalently used to couple with high-throughput sequencing on the Illumina platforms, resulting many successful applications for genome-wide genotyping, genetic mapping, quantitative trait locus (QTL) and association studies (Baird et al. 2008). However, RAD still depends on random fragmentations, reducing the consistence on SNP loci among samples. Elshire et.al subsequently developed a more straightforward genotyping method as GBS with restriction enzymes of ApeKI in maize and barley (Elshire et al. 2011). The protocols for GBS are simple, extremely specific and highly reproducible. In recent years, the easy transferability of GBS to other species leads to many application in plants (Poland \& Rife 2012). One of the most attracting features of GBS is the using of methylation-sensitive restriction enzymes during libraries constructions to avoid repetitive fragments and to simplify the reads alignments in extremely complex genomes (Elshire et al. 2011); therefore, GBS is an excellent whole-genome genotyping technique for complex non-model organism genomes with massive repetitive regions and abundant 
93

94 95

genetic diversities.

Teleost, representing a large portion of fish species, has been showed to undergo the third round of wholegenome duplication (WGD) 370 million years ago (Braasch et al. 2016; Xiao et al. 2015b). The extra WGD left a large portion of duplicated and repetitive sequences in teleost genomes (Berthelot et al. 2014; Jaillon et al. 2004), making the accurate whole-genome SNP marker development was still challenging in many teleost species (Wang et al. 2008). We speculated that GBS technique provided an efficient way and was suitable for genotyping in teleost complex genome. However, the whole-genome SNP development and association studies based on GBS is rarely reported on teleost fish species. Large yellow croaker (Larimichthys crocea), belonging to the Sciaenidae family of teleost, is an important marine fish in China and East Asia (Xiao et al. 2015a). Due to over-fishing and habitat degradation in last decades, the wild stock of the species has rapidly collapsed (Liu et al. 2008). The environmental changes and over-dense aquaculture pose more challenges on population conservation and sustainable development of the aquaculture for large yellow croaker. Whole-genome molecular markers and genome-wide association studies (GWAS) for important traits are prerequisites for the population conservation and genomic selection of the species (Steiner et al. 2013). However, the association studies are rarely reported for large yellow croaker, largely because of the lacking of abundant stable genomic SNP markers.

GBS technique provides the potential cost-efficient way for whole-genome SNP marker development in complex teleost genome. In the present investigation, we used large yellow croaker to verify the applicability of GBS on teleost. Two restriction enzymes of EcoRI and NlaIII based GBS protocol was developed and optimized. Massive whole-genome SNP markers were developed from the sequencing reads by bioinformatic pipelines, which were subsequently validated by Sequenom MassARRAY assay. The detected SNP markers in this work were then applied to the whole-genome association study of the muscle Eicosapentaenoic Acid (EPA) and Docosahexaenoic Acid (DHA) content in large yellow croaker. Our study confirmed the suitability of GBS on whole-genome SNP marker development in teleost genome. The developed whole-genome SNP markers and functional genes involved in muscle EPA and DHA contents offered valuable genetic resources for conservation genetics and genomic selection of large yellow croaker.

\section{Materials and Methods}

\section{Ethics Statement}


127 The sample collection and experiments in the study was approved by the Animal Care and Use Committee

128 of Fisheries College of Jimei University (Animal Ethics no. 1067).

129 Sample preparation and DNA extraction

130 The mixed reference population of 500 individuals was bred by a random fertilization of 30 males and 30 131 females at the large yellow croaker breeding base of Jimei University in Ningde, Fujian, China. All fish 132 individuals were 1.5 year old with the total length and weight of $24.5 \sim 25.9 \mathrm{~cm}$ and $217.8 \sim 234.1 \mathrm{~g}(95 \%$ 133 confidence interval), respectively. To extract genomic DNA respectively from 500 individuals, the dorsal fins $134(20-30 \mathrm{mg})$ of the fish individuals were collected, frozen in liquid nitrogen for the following DNA extraction. 135 Total genomic DNA was prepared in $1.5 \mathrm{ml}$ microcentrifuge tubes containing $550 \mu 1 \mathrm{TE}$ buffer $(100 \mathrm{mM} \mathrm{NaCl}$, $13610 \mathrm{mM}$ Tris, $\mathrm{pH} \mathrm{8,} 25 \mathrm{mM}$ EDTA, 0.5\% SDS and proteinase $\mathrm{K}, 0.1 \mathrm{mg} / \mathrm{ml}$ ). The samples were incubated at 55 $137{ }^{\circ} \mathrm{C}$ overnight and subsequently extracted twice using phenol and then phenol/chloroform (1:1) method. DNA 138 was precipitated by adding two and a half volumes of ethanol, collected by brief centrifugation, washed twice 139 with 70\% ethanol, air dried, re-dissolved in TE buffer (10 mM Tris-HCl, $1 \mathrm{mM}$ EDTA, pH 7.5). DNA 140 concentration and quality were estimated with an ND-1000 spectrophotometer (NanoDrop, Wilmington, DE, 141 USA) and by electrophoresis in $0.8 \%$ agarose gels with a lambda DNA standard.

\section{In silico enzyme assessment for GBS library construction}

143 To assess how endonucleases influence the DNA fragment length distribution, four enzyme combinations 144 were designed for in silico digestion of large yellow croaker genome: ApeKI-PstI, EcoRI-BstNI, EcoRI-NlaIII 145 and PstI-NlaIII (NEB, Ipswich, MA, USA). The activation and heat-inactivation temperature, location in 146 chromosome and length distribution of all fragments were analyzed to evaluation the best performance of each 147 enzyme combination.

\section{GBS library construction and sequencing}

149 The GBS libraries were constructed based on two DNA endonucleases: EcoRI (NEB, Ipswich, MA, USA) and NlaIII (NEB, Ipswich, MA, USA). A pilot GBS experiment was performed before the library construction to optimize the temperature and time parameters for yield, size distribution. Based on the pilot experiment, the GBS libraries of large yellow croaker based on EcoRI and NlaIII were constructed following the similar method in previous report (Beissinger et al. 2013). Briefly, genomic DNA $(20 \mathrm{ng} / \mu \mathrm{l})$ was incubated at $37^{\circ} \mathrm{C}$ with EcoRI and NlaIII, 10XCutSmart ${ }^{\mathrm{TM}}$ Buffer. The restriction reactions were heat-inactivated at $65^{\circ} \mathrm{C}$ by 20 min and were kept in $8^{\circ} \mathrm{C}$ for the following experiments. Sequencing adaptor and barcode mix, T4 DNA

156 Ligase, 10mM ATP and 10XCutSmart ${ }^{\mathrm{TM}}$ Buffer were incubated at $16^{\circ} \mathrm{C}$ for $2 \mathrm{~h}$ for ligation reactions. The 157 reactions were then heat-inactivated at $65^{\circ} \mathrm{C}$ by $20 \mathrm{~min}$ and the reaction systems were kept in $8^{\circ} \mathrm{C}$. Then, 158 polymerase chain reactions (PCR) experiments were performed in the reaction solutions $(20 \mu \mathrm{L})$ containing the 159 diluted restriction/ligation samples $(4 \mathrm{pM}, 2 \mu \mathrm{L})$, dNTP (each at $10 \mathrm{mM}, 5 \mu \mathrm{L})$, Taq DNA polymerase (NEB, 
160 Ipswich, MA, USA) (5 units $\mu \mathrm{L}, 0.25 \mu \mathrm{L}$ ), Illumina Primers (each at $10 \mu \mathrm{M}, 1 \mu \mathrm{L})$ and Indexing Primers $(10 \mu \mathrm{M}$,

$1611 \mu \mathrm{L}$ ). The PCR procedure was: $95^{\circ} \mathrm{C} 2 \mathrm{~min} ; 15$ cycle of $95^{\circ} \mathrm{C} 30 \mathrm{sec}, 60^{\circ} \mathrm{C} 30 \mathrm{sec}, 72^{\circ} \mathrm{C} 30 \mathrm{sec} ; 72^{\circ} \mathrm{C} 5 \mathrm{~min}$

162 and kept in $4{ }^{\circ} \mathrm{C}$. The PCR products were run on a $8 \%$ polyacrylamide gel electrophoresis. Fragments of

$163200 \sim 300 \mathrm{bp}$ were isolated using QIAGEN QIAquick ${ }^{\circledR}$ Gel Extraction Kit and diluted for pair-end sequencing

164 on an Illumina HiSeq 2500 sequencing platform (Illumina, Inc, San Diego, CA, USA).

\section{Sequencing read quality control and genotyping}

166 The raw sequencing reads generated by Illumina HiSeq 2500 from the GBS libraries were treated and 167 cleaned for SNP detection. First, the adaptors were removed and the resulted reads were split by sample168 specific barcode sequences. Only reads begins with the digest site sequences of EcoRI and NlaIII were retained 169 for the following quality control. Second, the overall base and read quality were accessed by FastQC. To avoid 170 the negative influence of ambiguous bases for SNP detection, reads with more than $5 \%$ of $\mathrm{N}$ were removed. 171 Then, the resulted reads were cleaned by the following steps: 1) discarding the reads that the quality lower than $17220 ; 2$ ) deleting 5bp windows in reads end that the average quality smaller than 20;3) removing read pairs if 173 one end was shorter than $50 \mathrm{bp}$.

174 The cleaned reads were mapped to large yellow croaker genome by BWA 0.7.6a (Li \& Durbin 2009). The 175 mapping was preceded by a short reads alignment with BWA-MEM algorithm. The alignment were then 176 sorted by coordinates and duplicate marked by SortSam and MarkDuplicates programs in Picard tools 1.107 177 (picard.sourceforge.net), respectively. To reduce the false positives of SNP detection in this study, three 178 processes were carried out: 1) short read mapping were re-aligned by local bases matches; 2) base Quality 179 Score Recalibration (BQSR) was employed to adjust the accuracy of the base and mapping quality scores; 3 ) 180 only reads pairs that both aligned on genome with a mapping score higher than 30 were used for SNP calling. 181 Then, the SNP markers were detected by GATK UnifiedGenotyper utility.

\section{SNP validation by Sequenom MassARRAY assay}

183 Genomic DNA was extracted from dorsal fin ray tissue as the method described before. PCR 184 amplification was performed in the reaction system ( $5 \mu$ l total volume) containing $20 \mathrm{ng}$ of genomic DNA, $1850.5 \mathrm{U}$ HotstarTaq (Qiagen), $0.5 \mu 1$ 10×PCR buffer, $0.1 \mu \mathrm{lNTPs}$ for each nucleotide and 0.5 pmol of each primer. 186 All PCR experiments were carried out in a PTC-100 PCR instrument (Eppendorf) with the following program: 1874 min denaturation at $94^{\circ} \mathrm{C}, 35$ cycles of $20 \mathrm{~s}$ at $94^{\circ} \mathrm{C}, 30 \mathrm{~s}$ at $56^{\circ} \mathrm{C}$ and $1 \mathrm{~min}$ at $72^{\circ} \mathrm{C}$ and a final extension at $18872^{\circ} \mathrm{C}$ for $3 \mathrm{~min}$. After the PCR products were cleaned using $2 \mu \mathrm{l}$ SAP (SEQUENOM), the single base extension 189 used $2 \mu 1$ EXTEND Mix (SEQUENOM) contained 0.94 $\mu$ l Extend primer Mix, 0.041 $\mu$ l iPLEX enzyme and $1900.2 \mu 1$ iPLEX termination mix and performed with the following steps: initial denaturation at $94^{\circ} \mathrm{C}$ for $30 \mathrm{~s}$, 191 followed by 40 cycles of 3-step amplification profile of $5 \mathrm{~s}$ at $94^{\circ} \mathrm{C}$, additional 5 cycles of $5 \mathrm{~s}$ at $52{ }^{\circ} \mathrm{C}$ and $5 \mathrm{~s}$ 192 at $80^{\circ} \mathrm{C}$ and a final extension at $72{ }^{\circ} \mathrm{C}$ for $3 \mathrm{~min}$. The PCR product was cleaned by resin purification and then 
193

194

195

196

197

198

199

200

201

202

203

204

205

206

207

208

209

210

211

212

213

214

215

216

217

218

219

220

221

222

223

analyzed using MassARRAY Analyzer Compac (SEQUENOM) and software TYPER (SEQUENOM).

To evaluate the accuracy of SNP detection in this study, the genotypes from GATK SNP calling were compared with those from MassARRAY assay. If the genotypes of one SNP locus from GATK calling were identical with that in MassARRAY, then the locus was called a correct genotype. As a result, 1,421 of 1500 SNP loci were correctly genotyped by GATK and the success rate of SNP calling was $\sim 94.7 \%$. The specificity and sensitivity of SNP calling in the study were also evaluated. The reference homozygous genotypes (AA) both from MassARRAY and GATK were called true negatives, and the heterozygous genotypes or allelic homozygous (AB and BB) both from MassARRAY and GATK were called true positives. Specificity was then calculated as the number of true positives divided by the number of true positives plus the number of false positives, and the sensitivity was estimated as the number of true positives divided by the number of true positives plus the number of false negatives as the following formula:

$$
\begin{aligned}
& \text { Specificity }=\frac{\text { true negative }}{\text { true negative }+ \text { false positive }} \\
& \text { Sensitivity }=\frac{\text { true positive }}{\text { true positive }+ \text { false negative }}
\end{aligned}
$$

\section{Association analysis with the muscle EPA and DHA content}

From the 500 large yellow croaker population, 200 individuals were randomly selected for the muscle EPA and DHA content measurement for the following statistics and association analysis. The fat acid composition analysis followed the similar methods in previous reports (Murillo et al. 2014). Briefly, the total lipid was extracted from the fresh muscle tissue using the chloroform-methanol method (Folch et al. 1957). After saponification with $1 \mathrm{ml}$ of $50 \% \mathrm{KOH}$ in $15 \mathrm{ml}$ ethanol, the lipid was then esterified in $80^{\circ} \mathrm{C}$ for $20 \mathrm{~min}$ using $6.7 \%$ boron trifluoride (BF3) in methanol (Morita chemical industries Co., Ltd., Osaka, Japan). After making up in hexane $(20 \mathrm{mg} / \mathrm{ml})$, fatty acid methyl esters (FAME) preparations were analyzed by gas chromatography (GC). The temperature increase of 170 to $260^{\circ} \mathrm{C}$ at $2{ }^{\circ} \mathrm{C} / \mathrm{min}$ was set and helium was used as the carrier gas. Since the muscle contents of EPA and DHA were highly correlated, we combined those two components together in fish muscle.

Prior to the association study, pairwise clustering based on the alleles shared identical by state (IBS) between any two individuals was performed to assess the genetic relatedness. The population stratification was analyzed by multi dimensional scaling (MDS) clustering methods available in the PLINK software (Purcell et al. 2007).

With the developed SNP markers, the association analysis was performed between genotypes and measured muscle EPA and DHA content using Plink 1.07. A simple linear regression of phenotype on genotype was performed in the analysis. Markers with p-values $\leq 1 \mathrm{e}-4$ were considered significantly associated 
224 with muscle EPA and DHA contents. To identify the biological functions of nearby genes and whether the 225 orthologs of these significantly associated loci were also associated with the EPA and DHA content in other 226 species, we identified the protein-coding genes around $50 \mathrm{~kb}$ of the significant SNP markers. We aligned the 227 genes against the NCBI nr database by Blastx (Altschul et al. 1997). GO term and KEGG pathway enrichment 228 analysis of the associated genes were performed with Gene set enrichment analysis (GSEA) (Shi \& Walker 229 2007) by two-tailed Fish's exact test with Benjamini \& Hochberg false discovery rate (FDR) (Benjamini \& 230 Hochberg 1995) against the background of the all protein-coding gene in large yellow croaker genome. The 231 additive genetic variances were estimated by using R-package EMMREML, Version 3.1. 232 (http://mirror.bjtu.edu.cn/cran/web/packages/EMMREML/index.html)

\section{Results}

\section{Enzyme assessment and GBS construction for large yellow croaker}

235 According to the principles of the enzyme combination design for GBS library construction, four enzyme combinations were designed for the GBS analysis of large yellow croaker genome: ApeKI-PstI, EcoRI-Bst $\mathrm{NI}$,

237 EcoRI-NlaIII and PstI-NlaIII (NEB, Ipswich, MA, USA). To assess the fragment size distribution and the 238 number of potential SNP marker developed, the public large yellow croaker draft genome sequences (Ao et al. 239 2015) were in silico digested by the four two-enzyme combinations to mimic the genomic fragmentation. As 240 shown in the Figure 1, the predicted fragment numbers decreased with the fragment size for all enzyme combinations, but the ApeKI-PstI and EcoRI-BstNI lead to a large portion of fragments longer than $1 \mathrm{~kb}$. According to the size distribution in Figure 1 and to make the fragment size more compatible to NGS sequencing, the genomic fragment with a size of 100 300 bp were preferred; therefore, EcoRI-NlaIII and PstINlaIII were the rational combinations for the following library construction. According to our in silico experiments, genomic fragment in the range of 200 300 bp were used to construct GBS libraries (See Method section for details). By the assessment of the combination of EcoRI-NlaIII, roughly 1.5 million fragments would be collected in libraries.

\section{Library sequencing and reads mapping}

GBS libraries were constructed by the two enzyme based digestion (see Method section for the details). The NGS sequencing of GBS libraries for 500 individuals generate roughly $314 \mathrm{~Gb}$ raw sequencing reads. To evaluate the raw data distribution among samples, we found that the majority of individuals $(\sim 95 \%)$ had the raw sequencing reads ranged from 600 to $650 \mathrm{Mb}$, indicating the excellent sequencing uniformity among samples from library construction and sequencing. The raw reads were cleaned by HTSeq to trim low quality ends (average quality $<20$ ) and eliminate short reads (length $<50 \mathrm{bp}$ ). The cleaned reads were mapped to large 
257 as an example (SI Figure 1). We found that reads were evenly covered all linkage groups of large yellow 258 croaker, indicating an ideal representativeness of the libraries at the whole genome level. The covered loci 259 depth distribution (SI Figure 2) showed that the majority of depth ranged from 5 to 20 and extreme reads 260 enrichment on genome local regions were successfully avoided in sequencing libraries.

\section{SNP discovery among samples in large yellow croaker genome}

262 To develop molecular markers based on the GBS library sequencing, SNP variants markers were detected 263 from the reads alignments by GATK (McKenna et al. 2010) pipelines (see Method and Material section for 264 detailed information). To improve the quality of the detected SNP, we employed the extra reads local re265 alignment and Base Quality Score Recalibration (BQSR) steps in SNP calling pipelines. Previous literatures on model organisms showed that those extra processes on reads alignment and SNP quality could significant reduce the false positives SNPs (DePristo et al. 2011; McKenna et al. 2010; Van der Auwera et al. 2002), therefore our refined bioinformatics pipeline coupled with the library construction provided a solid foundation for SNP detection in this study. As a result, 489,246 SNP markers were discovered in at least 200 large yellow croakers with a loci depth threshold of 3. It is not surprised that the majority of SNP markers were not shared by all samples because of the inherent DNA polymorphisms on enzyme digestion site in genome. The number of shared SNP markers among samples was crucial for the evaluation of the GBS sequencing of large yellow croaker genome, especially for the studies of QTL and GWAS analysis in populations. We further used depthand population-based method to investigate the influence of loci depth and population size on the number of shared SNP marker. As we expected, both the population size and loci depth dramatically influenced the number of shared SNP markers (Figure 2). However, hundreds of thousands of the shared SNP markers were identified with a depth threshold of 5 in the study. According to previous literatures on SNP development in non-model organisms, the depth filtering of 5 provided high quality SNP markers for the genetic studies (Hiremath et al. 2012; Nguyen et al. 2014); therefore, our SNP calling based on GBS library developed sufficient SNP markers for the biological trait mapping and conservation genetics. We indeed found the sharp decreases on the number of shared SNPs for the population size from 450 to 500 , which could be attributed to the samples with extremely low sequencing amount.

To control SNP marker quality while maximizing the number of shared samples and to facilitate the following GWAS analysis, markers with the loci depth higher than 5 and the shared in at least 400 individuals (90\% of all sample) were used for the following analysis, resulting 69,845 SNP markers in large yellow croaker genome. To answer the question if our sequencing data was sufficient for the whole-genome SNP development, the numbers of the detected genomic SNP markers were plotted against the sequencing data for 
291 distribution of those SNP markers in 24 linkage groups (Figure 4) showed that those SNP markers were ideally

292 evenly distributed in the genome, suggesting an excellent representation of whole genome markers in large

293 yellow croaker. The location and functions of SNPs were investigated by comparing the locus coordinates with

294 those of gene annotations. We found that $\sim 53 \%$ of these SNPs were from genic regions, including exons

295 (3,000), introns (27,114), and untranslated regions (UTRs, 9,166) (Figure 3). The detailed SNP categories in

296 UTR revealed that 4,311 and 4,855 SNPs were from 5UTR and 3UTR, respectively. The biological functions

297 of those SNP markers were analyzed according to their relative positions of the protein-coding genes. As in

298 Figure 5, 866 SNPs markers in coding regions caused synonymous mutations. Of the remaining markers, 3,022

299 SNPs could lead to a change of amino acid and introduction of frame shift and new or lost start/stop codons.

300 Those SNP markers might significantly alter the biological functions of the hosting genes and thus influence

301 the biological traits that controlled by those genes.

302 Experimental validation of detected SNP loci

303 To assess the reliability of the SNP makers developed from the reduced representation libraries, 50 loci

304 from 30 individuals were randomly selected to validate the marker polymorphism by the

305 Sequenom MassARRAY assay. As shown in Table 1, MassARRAY assay verified the most of detected SNP

306 markers in those samples. Among 1,500 markers, 1,421 were validated by MassARRAY, confirming our

307 library construction, sequencing and SNP marker calling pipelines. The primers for SNP validation and the

308 detailed genotypes were listed in SI Table 1 and SI Table 2, respectively. As shown in the Table 1, the

309 specificity and sensitivity for the SNP genotype detection in the present study were estimated as $94.2 \%$ and

$31098.3 \%$, respectively. Notably, we found that the majority of discordant genotypes were heterozygous, which

311 was consistent with the reports for other organisms (Sonah et al. 2013). We attributed the error-prone

312 genotypes in heterozygous markers to the fact that those markers need more supporting reads than their

313 homozygous counterparts. However, the Sequenom MassARRAY assay still successfully validated $\sim 95 \%$ of

314 the detected SNP marker developed by the GBS library sequencing, providing us solid SNP genotypes of the

315 following trait association and other genetics studies for large yellow croaker.

316 The association study with the muscle EPA and DHA content

317 To apply the genome-wide markers to probe potential marker and genes contributing to muscle EPA and

318 DHA contents, 200 large yellow croakers reared with the identical feed in the same netcage were used to

319 quantify EPA and DHA level. Muscle EPA and DHA contents in 176 individuals were successfully extracted

320 and measured. The contents exhibited a typical normal-like distribution (p-value of 0.94 with Kolmogorov-

321 Smirnov test) with an average of $21.5 \mathrm{mg} / \mathrm{g}$ and a standard deviation of $4.1 \mathrm{mg} / \mathrm{g}$ (SI Figure 3). The difference

322 of the highest and the lowest EPA and DHA contents was $\sim 13.8 \mathrm{mg} / \mathrm{g}$.

323 Before the association study, population stratification with MDS clustering showed that all samples were 
324 grouped into several clusters (SI Figure 4). 200 individuals used in the association study were distributed 325 among population clusters, providing diversified genetic background for the following studies. From the MDS 326 plot (SI Figure 4), the first and second dimension value (arbitrary value) were all smaller than 0.05 , indicating 327 homogeneous genotypes among samples. The association study of SNP marker with the muscle EPA and DHA 328 content was performed with the linear model with a covariance to sex in Plink (Purcell et al. 2007). 69,845 329 SNP loci developed above with depth threshold of 5 were used to perform the association study (Figure 5). As 330 shown in Figure 6, 39 markers from 11 linkage groups were exhibited significant association with the EPA and 331 DHA content ( $\mathrm{p}$-value $<1 \mathrm{e}-4)$. Notably, many associated markers were significant by clusters in linkage group 3324,5 and 11, suggesting the credibility of the association studies. The results might also imply that many genes 333 contributed to the muscle EPA and DHA levels in large yellow croaker. With the variance estimation by 334 Restricted Maximum Likelihood (REML) method (Smith \& Graser 1986), we found that those 39 significant 335 markers could interpret as high up to $\sim 63.0 \%$ of genetic variance explained by all 69,845 markers.

336 To identify gene contributing to the muscle EPA and DHA content in large yellow croaker, we 337 investigated the biological functions of protein-coding genes within $50 \mathrm{~kb}$ of all significant SNP markers (p338 value $<1 \mathrm{e}-4)$. As a result, 122 genes were identified from the above association regions. The biological KEGG 339 pathway and GO term annotations of the associated genes were enriched under the background of all protein340 coding genes. The metabolic pathway of fat digestion and absorption was significant $($ FDR $<0.023)$ in the 341 KEGG enrichment (Figure 6B, SI Table 3). Meanwhile, GO terms of unsaturated fatty acid biosynthetic 342 process, fatty acid derivative biosynthetic process and lipid transporter activity were also highlighted (FDR < 3430.05 ) for the associated functional genes (Figure 6C). The detailed gene function GO annotations were 344 summarized in SI table 4. We found that the many identified genes played important roles in lipid transport, 345 metabolism and transcription regulation, such as apolipoprotein $\mathrm{B}(A P O B)$, Carnitine O-acetyltrasferase 346 (CRAT) and oxysterol binding protein 10 (OSBPL10). APOB is a crucial lipid transport protein in organism. 347 Previous nutriology studies confirmed the correlation of EPA and DHA contents with APOB genotypes and 348 gene expression (Anil 2007). Given their close relationship, we speculated that the polymorphisms on $A P O B$ 349 gene might contribute to the EPA and DAH accumulation in large yellow croaker muscle. CRAT and 350 OSBPL10 may also involved in the muscle EPA and DHA content since carnitine and oxysterol were 351 important components and regulators in EPA and DHA synthesis pathways according to previous reports (Qiu 352 2003; Rise et al. 2002). Notably, we observed palmitoyl-protein thioesteraes 2 (PPT2) (around a maker with a 353 p-value of 6.7e-06) as a potential functional gene contributing to muscle EPA and DHA contents. PPT2 gene 354 was also identified by genome-wide association study on n-3 and n-6 polyunsaturated fatty acid levels in 355 Chinese and European-ancestry populations (Dorajoo et al. 2015; Hu et al. 2016). 
358

359

360

361

362

363

364

365

366

367

368

369

370

371

372

373

374

375

376

377

378

379

380

381

382

383

384

385

386

387

388

389

390

391

392

The advent and development of NGS have unprecedentedly prompted the application of the wholegenome marker development (Seeb et al. 2011). Recently, SNP developments on genomic level were performed in many species, including livestock and fish in agriculture (Sun et al. 2014). However, the cost for whole-genome re-sequencing is still one of the largest challenges in genomic marker developments. Based on NGS, GBS generally used multiple endonucleases to obtain the desired genomic length and the number of fragments to squeeze the sequencing cost (De Donato et al. 2013; Elshire et al. 2011; Sonah et al. 2013), thus improving the specificity of marker detection along individuals; however, most of the GBS application were reported for plant genomes. Teleost, representing a large portion of fish species, has been showed to undergo the additional third round of whole-genome duplication (WGD) 370 million years ago. The extra genome duplication led to a large portion of duplicated and repetitive sequences in teleost genomes (Sémon \& Wolfe 2007). GBS techniques provided an efficient way to probe polymorphism markers from complex genomes; however, the whole-genome SNP development and association studies based on GBS is rarely reported on teleost fish species. In this work, we used teleost large yellow croaker to verify the applicability of GBS on genomic marker development on teleost species. So far as we know, this is the first GBS implementation in large yellow croaker genome. The developed SNP markers provided useful resources for the following genetic studies, including population structure, conservation and functional gene mapping of important traits of the species. The enzyme combination and GBS protocols used in this study could also be valuable reference for other teleost species.

Our in silico experiments mimicked the two enzyme digestion on large yellow croaker genome. After the detail investigation of the enzymes, we chose the combination of EcoRI and NlaIII for GBS protocol for two reasons. Firstly, EcoRI and NlaIII possessed the identical heat-inactivation temperature, which facilitated the pilot studies to optimize the experimental conditions for library construction; secondly and more importantly, EcoRI was sensitive when restriction site overlaps methylation sequence of $\mathrm{CpG}$ islands, therefore the using of the enzyme would partially avoid the digestion in repetitive regions. Many previous GBS libraries were constructed with the fragment length 100 300 bp or even wider (Elshire et al. 2011; Sonah et al. 2013); however, we predicted $\sim 3$ million fragment would be generated in that range. The large number of fragment implied a large amount of sequencing reads to cover those genomic regions, which would increase the unitcost for the sequencing. To reduce the genomic fragments needed to be sequenced for libraries in this study, we attempted to narrow the length range to $200 \sim 300 \mathrm{bp}$, which was predicted to generate roughly 1.5 million genomic fragment for sequencing.

Taking the SNP frequency of 1 per 1000 bp (Pushkarev et al. 2009), the library sequencing might result into roughly 300 thousand SNP markers along large yellow croaker genome. Our estimation was based on the assumption that all individuals have no mutation on endonuclease digesting site and the read depth were high enough to cover SNP loci. However, because of the divergent genomic background among populations, it is very hard to detect all SNP markers that shared by all individuals. In this work, 489,246 raw SNP markers 
393 supported by more than three reads were detected with an average sequencing amount of $600 \mathrm{Mb}$ in at least

394200 individual from the 500 large yellow croaker population. To facilitate the following marker association

395 study and breeding practise, previous studies proposed several methods to filter the high quality SNP that

396 shared in more individuals, such as depth-based (Li et al. 2009), quality score-based (Brockman et al. 2008)

397 and population-based (Bansal et al. 2010) manner. In this study, we employed a composite strategy for SNP

398 filtering by simultaneously considering loci depth, marker quality and shared population size. As a result,

39969,845 SNP markers were left with a depth higher than 5, quality score higher than 100 and shared with at

400 least $80 \%$ individuals (400 large yellow croakers). More than half ( $\sim 53 \%)$ of those detected quality SNP

401 markers resided in genic regions, enabling us to probe the possible association of trait with the nearby

402 functional genes. We noticed that the percentage of markers in genic regions was higher than that of previous

403 reports in soybean (39.5\%) (Sonah et al. 2013) but lower than that of sweat cherry (65.6\%) (Guajardo et al.

404 2015). Those SNP markers generated from the reduced representation library, especially those in genic regions,

405 provided us an easy and efficient manner to detect genomic small variants and to identify genomic regions

406 related to important traits of large yellow croaker at genomic scale. The detected SNP markers were then

407 validated by Sequenom MassARRAY assay for the randomly selected 50 loci in 30 individuals. Although the

408 success rate (94.6\%) was slight lower than that reported in the similar study in soybean (98\%) (Sonah et al.

409 2013), the library preparation protocol and bioinformatics pipeline provided us high quality genotypes on those

410 SNP loci in the population for the following association studies.

411 The successful applications of GWAS have greatly prompted the understanding to the genetic bases of

412 important economic traits and would eventually benefit the artificial breeding and population conservation of

413 non-model species (Correa et al. 2015; Narum et al. 2013). EPA and DHA are both omega-3 poly-unsaturated

414 fatty acids that important in human physiology (Swanson et al. 2012). Previous medical experiments

415 demonstrated their positive effects on depressive symptoms in clinical trials (Hoffmire et al. 2012) and the

416 essential functions in brain development (Brenna \& Carlson 2014). Marine fish is a main source for human

417 EPA and DHA supplement and nutritional properties of fish meat are highly dependent on polyunsaturated

418 fatty acid levels; therefore the EPA and DHA content in muscle is one of the important indicants for the meat

419 quality of fish. The genetic bases controlling EPA and DHA accumulation in fish species are highly

420 interconnected and not fully revealed. Identifying key SNP loci and functional genes will increase our

421 knowledge of molecular mechanism of polyunsaturated fatty acid synthesis and metabolism in marine fish. To

422 the best of our knowledge, most of the previous researches were focus on the genetic variants on poly

423 unsaturated fatty acid metabolism after fish oil supplements in human or gene expression and EPA and DHA

424 level changes with different feed in fish (Gregory et al. 2016; Li et al. 2014; Li et al. 2013; Trushenski et al.

425 2012). The association studies aiming to identity potential functional genes contributing to EPA and DHA

426 accumulation in fish meat is rarely reported.

427 Among 176 individuals that were used to measure the muscle EPA and DHA level, the average muscle 
428 EPA and DHA content in the top 20 large yellow croakers $(28.4 \mathrm{mg} / \mathrm{g})$ was almost two-fold of that in the 429 lowest 20 ones $(14.6 \mathrm{mg} / \mathrm{g})$. Given that those fish were reared in the same cage and fed with the identical feed, 430 there was a great potential to raise the muscle EPA and DHA content in large yellow croaker via genetic 431 improvements. Using the developed quality SNP markers by GBS protocol, 39 SNP markers from 11 linkage 432 groups were observed to be significantly associated with muscle EPA and DHA levels. From the coordinates 433 of gene and SNP loci, 122 protein-coding genes were identified around those significant markers. The 434 functional analysis by homological searching found that many genes were involved in fat metabolism and 435 transport, such as $A P O B, C R A T$ and OSBPL10. Unsaturated fatty acid biosynthetic process, fatty acid 436 derivative biosynthetic process and lipid transporter activity and fat digestion and absorption pathway were 437 significantly enriched in GO terms and KEGG pathways for the identified functional genes. Meanwhile, we 438 observed large numbers of genes functions in cellular metabolism, gene expression and translation regulation, 439 which may also play a role in modulating muscle EPA and DHA contents (SI Table 2 and 3). Interestingly, we 440 identified the potential functional gene of $P P T 2$ gene in large yellow croaker that was previously discovered 441 during the whole-genome association of plasma n-3 and n-6 polyunsaturated fatty acid level in Asian and 442 European populations (Hu et al. 2016). The PPT2 gene in the linkage group 5 of large yellow croaker might 443 play a similar function in human and also contribute to the muscle EPA and DHA level. This result suggested 444 that teleost fish and human may shared similar metabolic pathway for the polyunsaturated fatty acid synthesis 445 and accumulation; however biological functions of PPT2 gene for the muscle EPA and DHA content in large 446 yellow croaker and other vertebrates need further gene functional analysis.

\section{Conclusions}

Teleost were widely believed to undergo the third round of WGD during the natural evolution; therefore, genomes of many teleost species were characterized by the complexity of high heterozygosity and repeat contents. In this work, EcoRI-NlaIII based GBS protocol was used to develop the whole-genome SNP markers in teleost large yellow croaker. The study verified the applicability of GBS on teleost species and provided useful references for GBS applications in other fish species. For large yellow croaker, about 70,000 high quality SNP markers, supported by at least 400 individuals in population, were detected from the GBS libraries. Those SNP markers were further experimentally validated by Sequenom MassARRAY assay. The even distribution and diversified biological impacts of those molecular makers confirmed the effect and efficiency of the GBS-based SNP development in large yellow croaker. With muscle EPA and DHA contents from 176 and 122 significantly associated SNP loci and related protein-coding genes were identified. The functional analysis of the related genes confirmed the results of the association study.

461 For the aspect of molecular resources, our developed SNP markers could be valuable genetic resources 
462 for large yellow croaker, and would be used in the following population structure, conservation genetics and 463 the association studies for other important economic traits. The associated results for the muscle EPA and 464 DHA content, namely the significant SNP loci and functional genes, provided us important guidance for the 465 further investigation of genetic bases of the muscle EPA and DHA accumulation in large yellow croaker and 466 would eventually aid the technological development towards the genetic improvement of meat quality via the 467 molecular-aided selection of the species.

468

469

470

471

472

473

474

475

476

477 Tables and Figure legends

478 Tables

479 Table1: SNP validation by Sequenom MassARRAY assay. NNs indicate the failed genotypes during the 480 SNP filtering.

\begin{tabular}{|c|c|c|c|c|c|}
\hline \multirow{2}{*}{\multicolumn{2}{|c|}{ genotypes }} & \multicolumn{4}{|c|}{ Sequenom MassARRAY assay } \\
\hline & & AA & $\mathrm{AB}$ & $\mathrm{BB}$ & $\mathrm{NN}$ \\
\hline \multirow{4}{*}{ 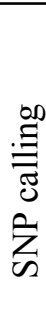 } & $\mathrm{AA}$ & 901 & 0 & 2 & 0 \\
\hline & $\mathrm{AB}$ & 54 & 404 & 7 & 0 \\
\hline & BB & 0 & 2 & 116 & 0 \\
\hline & NN & 11 & 1 & 2 & 0 \\
\hline
\end{tabular}


481

482 Figure legends

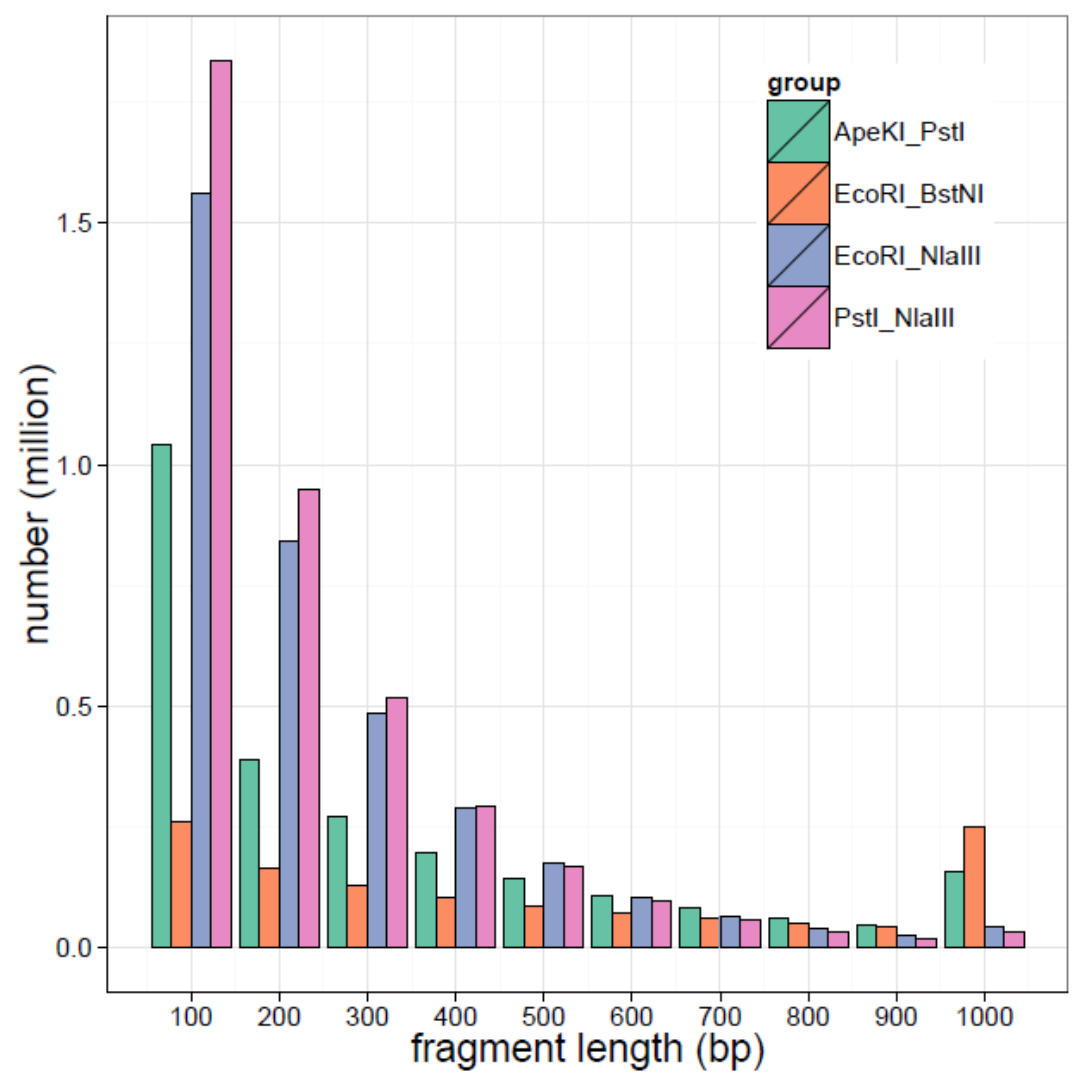

484 Figure 1: Fragment length distribution by restraint enzyme combination. Note that all fragments longer 485 than $1 \mathrm{~kb}$ were accumulated in the last bar. 


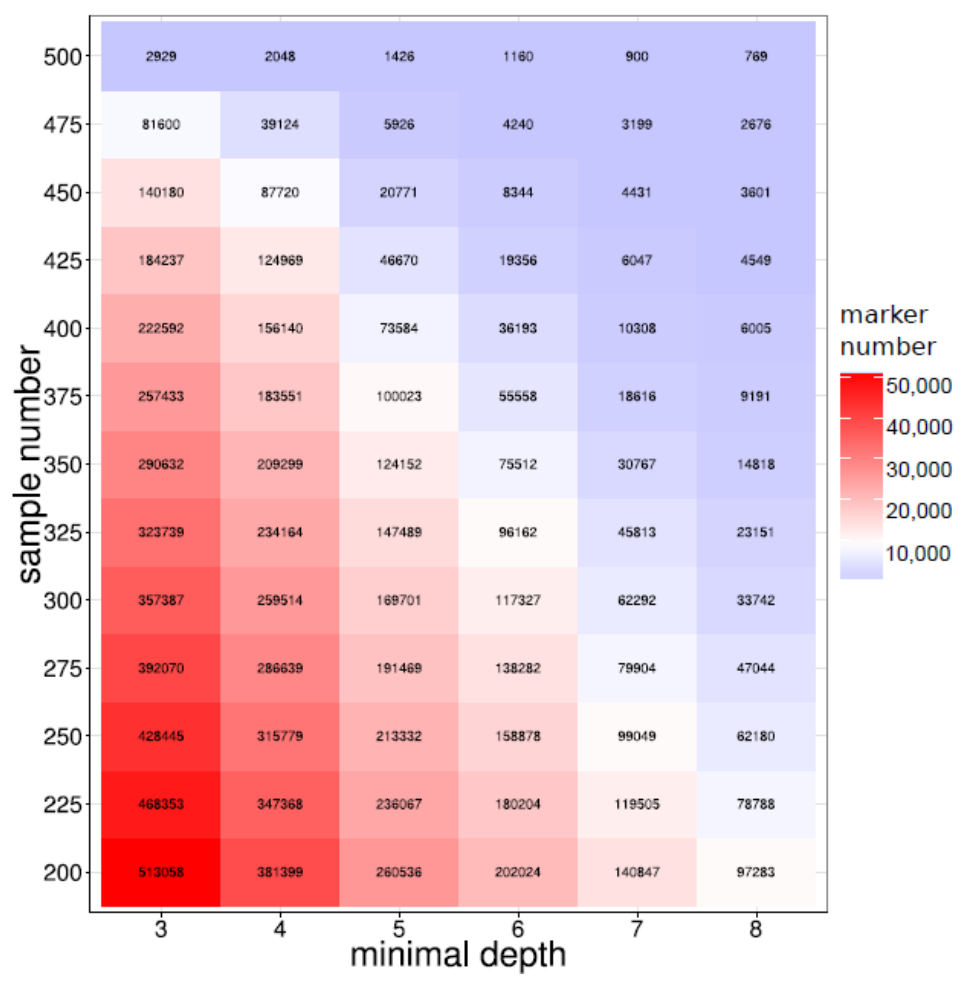

487 Figure 2: SNP number against sequencing depth and completeness. Note that only SNP loci with a quality 488 score larger than 100 were used in the analysis.

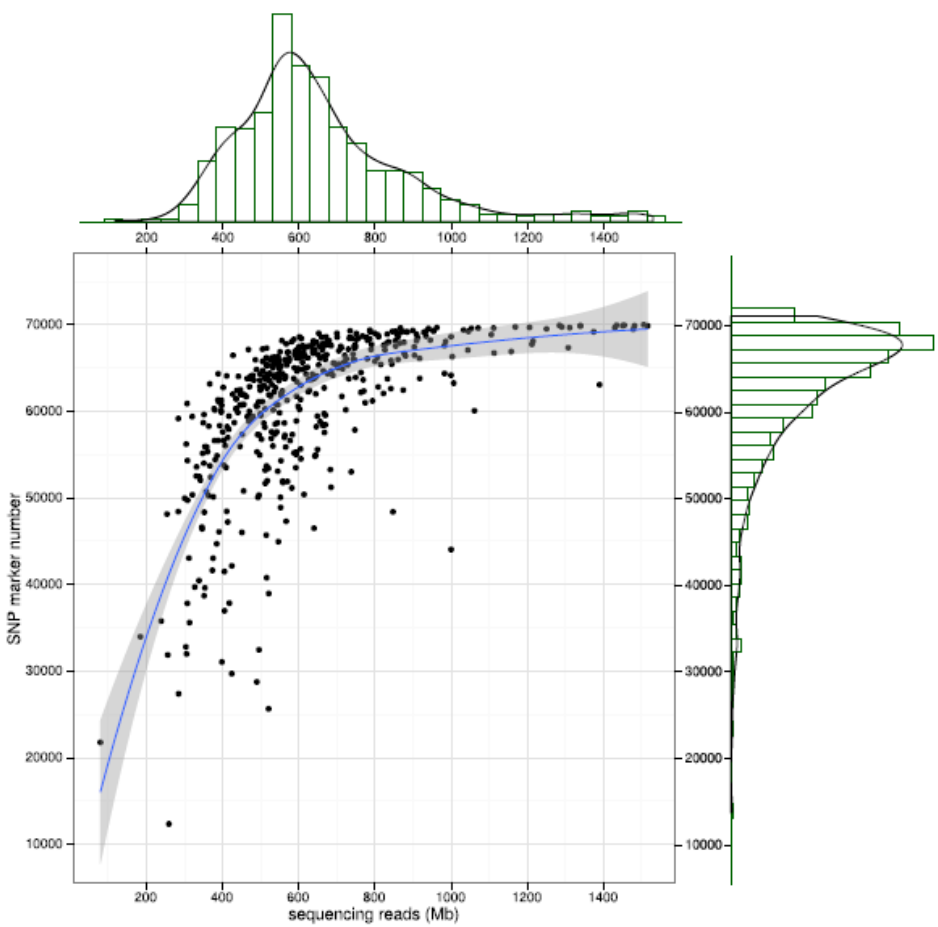

490 Figure 3: SNP number against sequencing amount. The distribution of sequencingamount (top) and SNP 
491 marker number (right) were plot by sides. The line in the scatter is the smoothed curve cross all samples, and

492 the grey area represent the $95 \%$ of the confidence region.

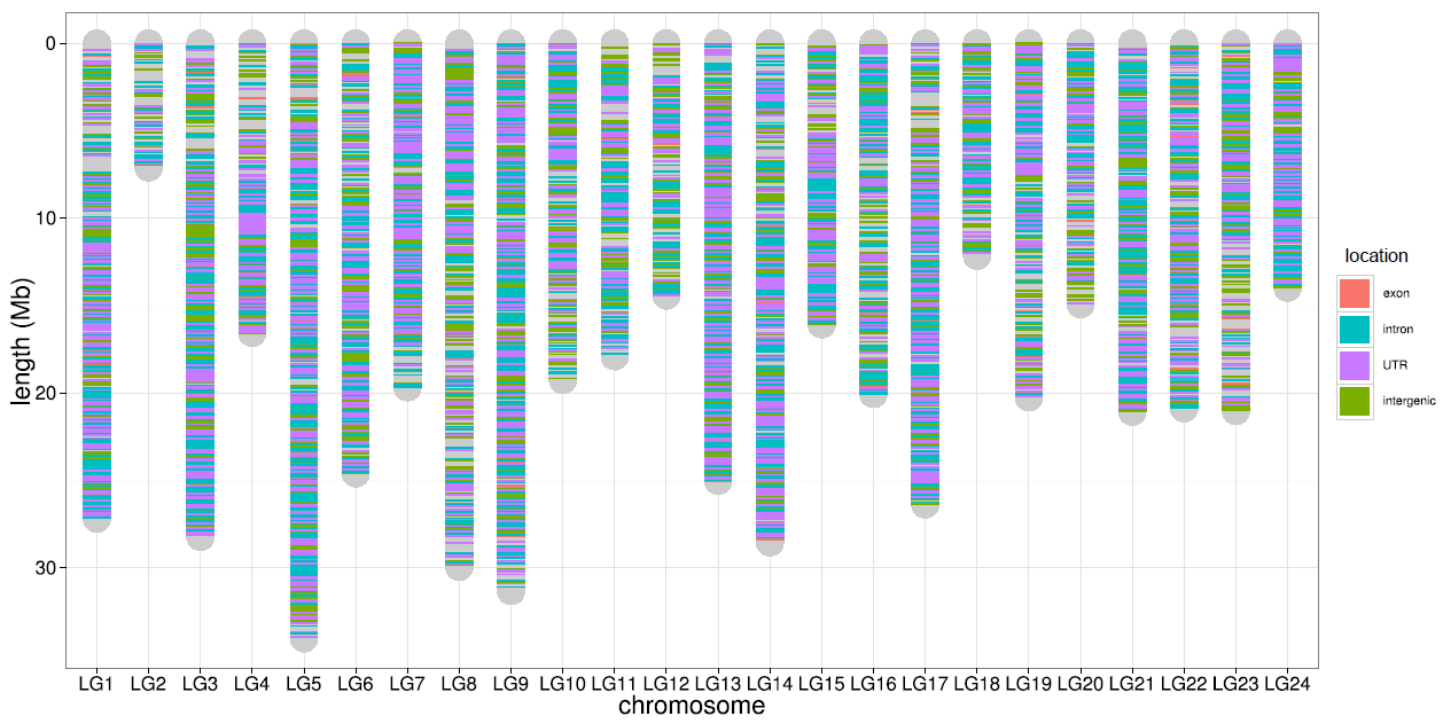

494 Figure 4: SNP distribution along chromosome. The lines along the chromosomes represent SNP loci. The 495 SNP location in exon, intron, UTR and intergenic regions are showed by red, blue, purple and green, 496 respectively.

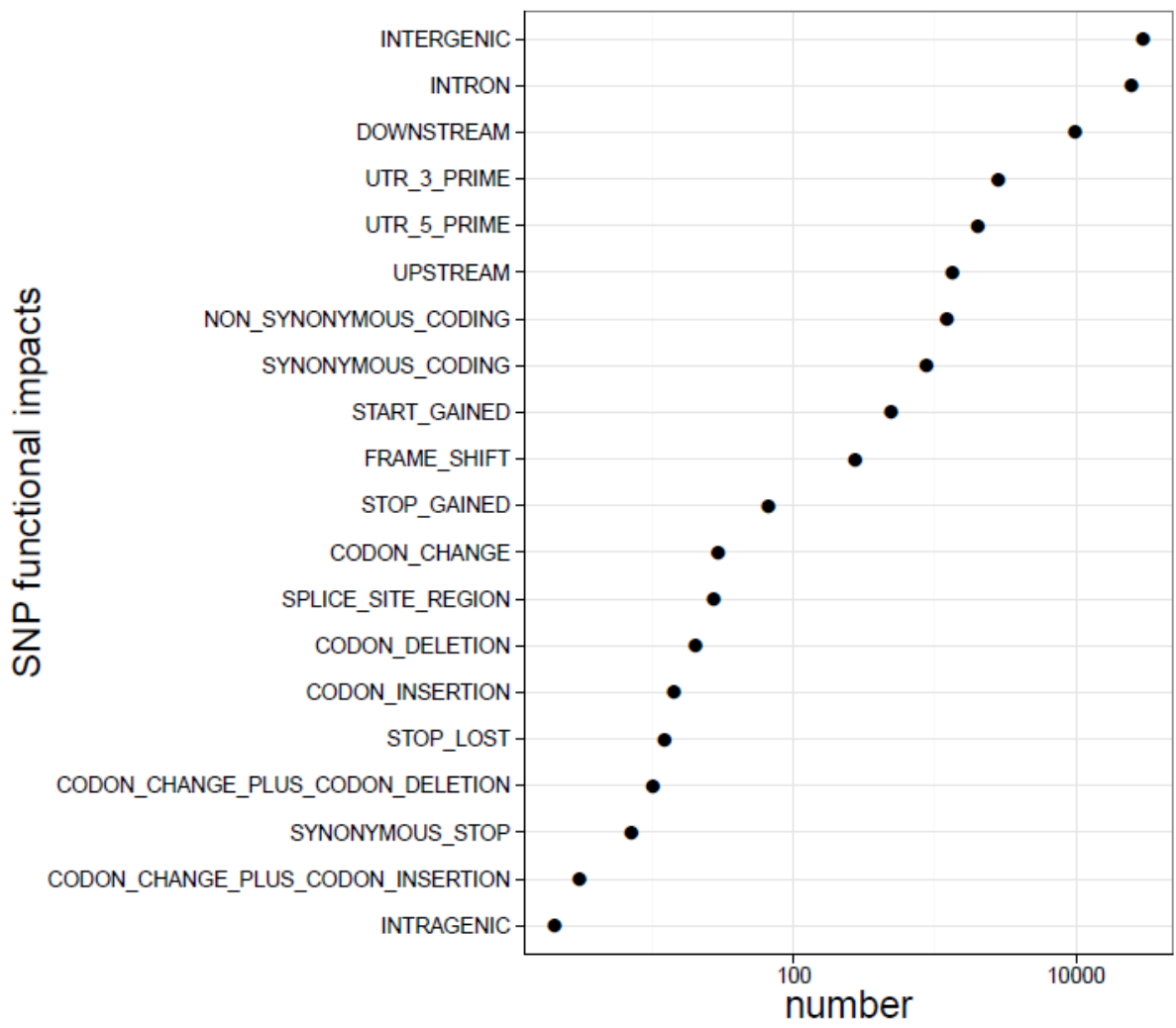


498 Figure 5: Biological impact annotations of high quality SNP markers that shared by at least $80 \%$ of the 499 population with 500 large yellow croakers.

500

501

502

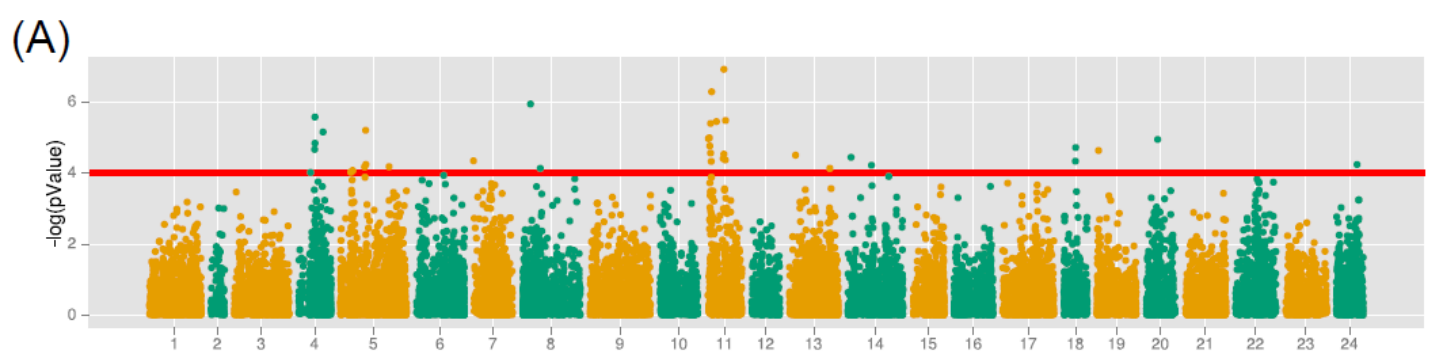

(B)

503

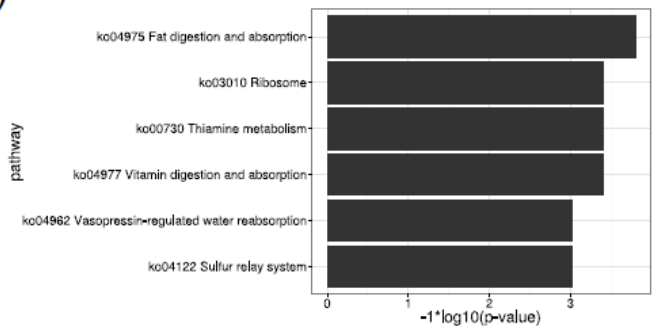

(C)

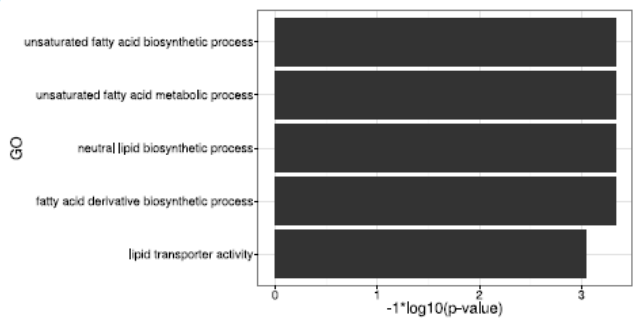

504 Figure 6: GWAS analysis on the muscle EPA and DHA content and the functional analysis therelated protein-coding genes. (A) The association results were illuminated in the Manhattan plot. The red line is the p-value threshold for significant markers; (B) KEGG pathway enrichment of functional genes; (C) GO term enrichment of related biological functions for the associated genes.

508

SI Tables and Figures legends

\section{SI Tables}

511 SI Table 1: Primers used for SNP validation in Sequenom MassARRAY assay.

512 SI Table 2: The detailed genotypes that called from GATK and Sequenom MassARRAY assay for 513 randomly selected 50 loci in 30 samples. The genotypes from GATK calling (AA) and MassARRAY (BB) 514 were listed together as AA/BB in each cell.

515 SI Table 3: KEGG pathway enrichment results for the functional genes associated with the muscle EPA and DHA content. The explanation of columns are: the first column of is the GO or KEGG Id; Pvalue is the 
517 p-value calculated from enrichment analysis; OddsRatio is the odds ratio from the enrichment analysis;

518 ExpCount is the expected gene count; Count is the real gene count in data; Size is the total genes assigned to

519 this term; Term is the biological description of term; FDR is the false discovery rate calculated from p-values.

520 SI Table 4: GO term enrichment results for the functional genes associated with the muscle EPA and

521 DHA content. Cell component (CC), Molecular Function (MF) and Biological Process (BP) were included in

522 theseparated excel sheet. The meanings of the columns are identical with SI Table 3.

523 SI Figure Legends

524 SI Figure 1: reads distribution along chromosomes for sample 88.

525 SI Figure 2: reads depth distribution in the library sequencing for sample 88.

526 SI Figure 3: EPA and DHA contents distribution.

527 SI Figure 4: Population stratification for all samples (black) and individuals (red) used in the association 528 analysis.

529

530

Data Accessibility

531

532

533

534

535

536

537

538

539

540

541

542

543

544

545

546

The sequencing short reads were deposited in the NCBI Sequence Read Archive (SRA) under project accession number of PRJNA309464.

\section{Acknowledgements}

We thanks the help of the stuffs in the large yellow croaker breeding base of Jimei University.

\section{References}

Altschul SF, Madden TL, Schäffer AA, Zhang J, Zhang Z, Miller W, and Lipman DJ. 1997. Gapped BLAST and PSI-BLAST: a new generation of protein database search programs. Nucleic acids research 25:3389-3402.

Anil E. 2007. The impact of EPA and DHA on blood lipids and lipoprotein metabolism: influence of apoE genotype. Proceedings of the Nutrition Society 66:60-68.

Ao J, Mu Y, Xiang LX, Fan D, Feng M, Zhang S, Shi Q, Zhu LY, Li T, and Ding Y. 2015. Genome sequencing of the perciform fish Larimichthys crocea provides insights into molecular and genetic mechanisms of stress adaptation. PLoS genetics 11:e1005118.

Baird NA, Etter PD, Atwood TS, Currey MC, Shiver AL, Lewis ZA, Selker EU, Cresko WA, and Johnson EA. 2008. Rapid SNP discovery and genetic mapping using sequenced RAD markers. PLoS One 3:e3376. 
547 Bansal V, Harismendy O, Tewhey R, Murray SS, Schork NJ, Topol EJ, and Frazer KA. 2010. Accurate

548

549

550

551

552

553

554

555

556

557

558

559

560

561

562

563

564

565

566

567

568

569

570

571

572

573

574

575

576

577

578

579

580

581

582

583

584

585 detection and genotyping of SNPs utilizing population sequencing data. Genome research 20:537-545.

Beissinger TM, Hirsch CN, Sekhon RS, Foerster JM, Johnson JM, Muttoni G, Vaillancourt B, Buell CR, Kaeppler SM, and de Leon N. 2013. Marker density and read depth for genotyping populations using genotyping-by-sequencing. Genetics 193:1073-1081.

Benjamini Y, and Hochberg Y. 1995. Controlling the false discovery rate: a practical and powerful approach to multiple testing. Journal of the Royal Statistical Society Series B (Methodological) 57:289-300.

Berthelot C, Brunet F, Chalopin D, Juanchich A, Bernard M, Noël B, Bento P, Da Silva C, Labadie K, and Alberti A. 2014. The rainbow trout genome provides novel insights into evolution after whole-genome duplication in vertebrates. Nature communications 5:3657-3657.

Braasch I, Gehrke AR, Smith JJ, Kawasaki K, Manousaki T, Pasquier J, Amores A, Desvignes T, Batzel P, and Catchen J. 2016. The spotted gar genome illuminates vertebrate evolution and facilitates humanteleost comparisons. Nature genetics 48:427-437.

Brenna JT, and Carlson SE. 2014. Docosahexaenoic acid and human brain development: Evidence that a dietary supply is needed for optimal development. Journal of human evolution 77:99-106.

Brockman W, Alvarez P, Young S, Garber M, Giannoukos G, Lee WL, Russ C, Lander ES, Nusbaum C, and Jaffe DB. 2008. Quality scores and SNP detection in sequencing-by-synthesis systems. Genome research 18:763-770.

Correa K, Lhorente JP, López ME, Bassini L, Naswa S, Deeb N, Di Genova A, Maass A, Davidson WS, and Yáñez JM. 2015. Genome-wide association analysis reveals loci associated with resistance against Piscirickettsia salmonis in two Atlantic salmon (Salmo salar L.) chromosomes. BMC genomics 16:1-9.

De Donato M, Peters SO, Mitchell SE, Hussain T, and Imumorin IG. 2013. Genotyping-by-sequencing (GBS): a novel, efficient and cost-effective genotyping method for cattle using next-generation sequencing. PLoS One 8:e62137.

De Pristo MA, Banks E, Poplin R, Garimella KV, Maguire JR, Hartl C, Philippakis AA, Del Angel G, Rivas MA, and Hanna M. 2011. A framework for variation discovery and genotyping using next-generation DNA sequencing data. Nature genetics 43:491-498.

Dorajoo R, Sun Y, Han Y, Ke T, Burger A, Chang X, Low HQ, Guan W, Lemaitre RN, and Khor C-C. 2015. A genome-wide association study of n-3 and n-6 plasma fatty acids in a Singaporean Chinese population. Genes \& nutrition 10:1-11.

Elshire RJ, Glaubitz JC, Sun Q, Poland JA, Kawamoto K, Buckler ES, and Mitchell SE. 2011. A robust, simple genotyping-by-sequencing (GBS) approach for high diversity species. PLoS One 6:e19379.

Folch J, Lees M, and Sloane-Stanley G. 1957. A simple method for the isolation and purification of total lipids from animal tissues. Journal of biological chemistry 226:497-509.

Gregory M, Collins R, Tocher D, James M, and Turchini G. 2016. Nutritional regulation of long-chain PUFA biosynthetic genes in rainbow trout (Oncorhynchus mykiss). The British journal of nutrition:115:1-9.

Guajardo V, Solís S, Sagredo B, Gainza F, Muñoz C, Gasic K, and Hinrichsen P. 2015. Construction of high density sweet cherry (Prunus avium L.) linkage maps using microsatellite markers and SNPs detected by genotyping-by-sequencing (GBS). PLoS One 10:e127750. 
586 Hiremath PJ, Kumar A, Penmetsa RV, Farmer A, Schlueter JA, Chamarthi SK, Whaley AM,

587

588

589

590

591

592

593

594

595

596

597

598

599

600

601

602

603

604

605

606

607

608

609

610

611

612

613

614

615

616

617

618

619

620

621

622

623

624

Carrasquilla - Garcia N, Gaur PM, and Upadhyaya HD. 2012. Large-scale development of costeffective SNP marker assays for diversity assessment and genetic mapping in chickpea and comparative mapping in legumes. Plant biotechnology journal 10:716-732.

Hoffmire CA, Block RC, Thevenet-Morrison K, and van Wijngaarden E. 2012. Associations between omega-3 poly-unsaturated fatty acids from fish consumption and severity of depressive symptoms: an analysis of the 2005-2008 National Health and Nutrition Examination Survey. Prostaglandins, leukotrienes and essential fatty acids 86:155-160.

Hu Y, Lu L, Manichaikul A, Zhu J, Chen Y-DI, Sun L, Liang S, Siscovick DS, Steffen LM, and Tsai MY. 2016. Genome-wide meta-analyses identify novel loci associated with n-3 and n-6 polyunsaturated fatty acid levels in Chinese and European-ancestry populations. Human molecular genetics:ddw002.

Jaillon O, Aury J-M, Brunet F, Petit J-L, Stange-Thomann N, Mauceli E, Bouneau L, Fischer C, Ozouf-Costaz C, and Bernot A. 2004. Genome duplication in the teleost fish Tetraodon nigroviridis reveals the early vertebrate proto-karyotype. Nature 431:946-957.

Li H, and Durbin R. 2009. Fast and accurate short read alignment with Burrows-Wheeler transform. Bioinformatics 25:1754-1760.

Li H, Liu J-P, Zhang M-L, Yu N, Li E-C, Chen L-Q, and Du Z-Y. 2014. Comparative Analysis of Fatty Acid Profiles in Brains and Eyes of Five Economic Fish Species in Winter and Summer. Journal of Food and Nutrition Research 2:722-730.

Li Q, Ai Q, Mai K, Xu W, and Zheng Y. 2013. A comparative study: In vitro effects of EPA and DHA on immune functions of head-kidney macrophages isolated from large yellow croaker (Larmichthys crocea). Fish \& shellfish immunology 35:933-940.

Li R, Li Y, Fang X, Yang H, Wang J, Kristiansen K, and Wang J. 2009. SNP detection for massively parallel whole-genome resequencing. Genome research 19:1124-1132.

Lipshutz RJ, Fodor SP, Gingeras TR, and Lockhart DJ. 1999. High density synthetic oligonucleotide arrays. Nature genetics 21:20-24.

Liu M, Mitcheson D, and Sadovy Y. 2008. Profile of a fishery collapse: why mariculture failed to save the large yellow croaker. Fish and Fisheries 9:219-242.

McKenna A, Hanna M, Banks E, Sivachenko A, Cibulskis K, Kernytsky A, Garimella K, Altshuler D, Gabriel S, and Daly M. 2010. The Genome Analysis Toolkit: a MapReduce framework for analyzing nextgeneration DNA sequencing data. Genome research 20:1297-1303.

Miller MR, Dunham JP, Amores A, Cresko WA, and Johnson EA. 2007. Rapid and cost-effective polymorphism identification and genotyping using restriction site associated DNA (RAD) markers. Genome research 17:240-248.

Muir P, Li S, Lou S, Wang D, Spakowicz DJ, Salichos L, Zhang J, Weinstock GM, Isaacs F, and Rozowsky J. 2016. The real cost of sequencing: scaling computation to keep pace with data generation. Genome Biology 17:1-9.

Murillo E, Rao K, and Durant AA. 2014. The lipid content and fatty acid composition of four eastern central Pacific native fish species. Journal of Food Composition and Analysis 33:1-5. 
625

626

627

628

629

630

631

632

633

634

635

636

637

638

639

640

641

642

643

644

645

646

647

648

649

650

651

652

653

654

655

656

657

658

659

660

661

662

663

Narum SR, Buerkle CA, Davey JW, Miller MR, and Hohenlohe PA. 2013. Genotyping - by - sequencing in ecological and conservation genomics. Molecular Ecology 22:2841-2847.

Nguyen TTT, Hayes BJ, and Ingram BA. 2014. Genetic parameters and response to selection in blue mussel (Mytilus galloprovincialis) using a SNP-based pedigree. Aquaculture 420-421:295-301.

Poland JA, and Rife TW. 2012. Genotyping-by-sequencing for plant breeding and genetics. The Plant Genome 5:92-102.

Popova T, Boeva V, Manie E, Rozenholc Y, Barillot E, and Stern M-H. 2013. Analysis of Somatic Alterations in Cancer Genome: From SNP Arrays to Next Generation Sequencing. Sequence and Genome Analysis I-Humans, Animals and Plants. 204:63-97

Purcell S, Neale B, Todd-Brown K, Thomas L, Ferreira MA, Bender D, Maller J, Sklar P, De Bakker PI, and Daly MJ. 2007. PLINK: a tool set for whole-genome association and population-based linkage analyses. The American Journal of Human Genetics 81:559-575.

Pushkarev D, Neff NF, and Quake SR. 2009. Single-molecule sequencing of an individual human genome. Nature biotechnology 27:847-850.

Qiu X. 2003. Biosynthesis of docosahexaenoic acid (DHA, 22: 6-4, 7, 10, 13, 16, 19): two distinct pathways. Prostaglandins, leukotrienes and essential fatty acids 68:181-186.

Rise P, Marangoni F, and Galli C. 2002. Regulation of PUFA metabolism: pharmacological and toxicological aspects. Prostaglandins, leukotrienes and essential fatty acids 67:85-89.

Rowe H, Renaut S, and Guggisberg A. 2011. RAD in the realm of next-generation sequencing technologies. Molecular Ecology 20:3499-3502.

Sémon M, and Wolfe KH. 2007. Rearrangement rate following the whole-genome duplication in teleosts. Molecular biology and evolution 24:860-867.

Seeb J, Carvalho G, Hauser L, Naish K, Roberts S, and Seeb L. 2011. Single-nucleotide polymorphism (SNP) discovery and applications of SNP genotyping in nonmodel organisms. Molecular Ecology Resources $11: 1-8$.

Shi J, and Walker MG. 2007. Gene set enrichment analysis (GSEA) for interpreting gene expression profiles. Current Bioinformatics 2:133-137.

Smith S, and Graser H-U. 1986. Estimating variance components in a class of mixed models by restricted maximum likelihood. Journal of Dairy Science 69:1156-1165.

Sonah H, Bastien M, Iquira E, Tardivel A, Légaré G, Boyle B, Normandeau É, Laroche J, Larose S, and Jean M. 2013. An improved genotyping by sequencing (GBS) approach offering increased versatility and efficiency of SNP discovery and genotyping. PLoS One 8:e54603.

Steiner CC, Putnam AS, Hoeck PE, and Ryder OA. 2013. Conservation genomics of threatened animal species. Annual review of animal biosciences 1:261-281.

Sun L, Liu S, Wang R, Jiang Y, Zhang Y, Zhang J, Bao L, Kaltenboeck L, Dunham R, and Waldbieser G. 2014. Identification and analysis of genome-wide SNPs provide insight into signatures of selection and domestication in channel catfish (Ictalurus punctatus). PLoS One 9:e109666.

Swanson D, Block R, and Mousa SA. 2012. Omega-3 fatty acids EPA and DHA: health benefits throughout life. Advances in Nutrition: An International Review Journal 3:1-7.

Peer] reviewing PDF | (2016:06:11154:1:0:NEW 5 Oct 2016) 
664 Trushenski J, Schwarz M, Bergman A, Rombenso A, and Delbos B. 2012. DHA is essential, EPA appears

665 largely expendable, in meeting the $\mathrm{n}$ - 3 long-chain polyunsaturated fatty acid requirements of juvenile 666 cobia Rachycentron canadum. Aquaculture 326:81-89.

667 Van der Auwera GA, Carneiro MO, Hartl C, Poplin R, del Angel G, Levy-Moonshine A, Jordan T, Shakir K, 668 Roazen D, Thibault J, Banks E, Garimella KV, Altshuler D, Gabriel S, and DePristo MA. 2002. From FastQ Data to High-Confidence Variant Calls: The Genome Analysis Toolkit Best Practices Pipeline. Current Protocols in Bioinformatics 11:11

Wang S, Meyer E, McKay JK, and Matz MV. 2012. 2b-RAD: a simple and flexible method for genome-wide genotyping. Nature methods 9:808-810.

Wang S, Sha Z, Sonstegard TS, Liu H, Xu P, Somridhivej B, Peatman E, Kucuktas H, and Liu Z. 2008.

675 Xiao S, Han Z, Wang P, Han F, Liu Y, Li J, and Wang ZY. 2015a. Functional marker detection and analysis

676

677 on a comprehensive transcriptome of large yellow croaker by next generation sequencing. PLoS One

678 10:e124432.

679

Xiao S, Wang P, Zhang Y, Fang L, Liu Y, Li JT, and Wang ZY. 2015b. Gene map of large yellow croaker

680 (Larimichthys crocea) provides insights into teleost genome evolution and conserved regions

681 associated with growth. Scientific reports 5: 18661.

682

683

684 


\section{Figure 1 (on next page)}

Fragment length distribution by restraint enzyme combination. Note that all fragments longer than $1 \mathrm{~kb}$ were accumulated in the last bar.

Note that all fragments longer than $1 \mathrm{~kb}$ were accumulated in the last bar. 


\section{Figure 2 (on next page)}

SNP number against sequencing depth and completeness. Note that only SNP loci witha quality score larger than 100 were used in the analysis.

Note that only SNP loci witha quality score larger than 100 were used in the analysis. 


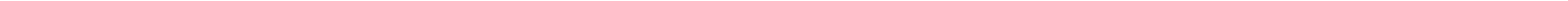


Figure 3 (on next page)

SNP number against sequencing amount.

SNP number against sequencing amount. The distribution of sequencingamount (top) and SNP marker number (right) were plot by sides. The line in the scatter is the smoothed curve cross all samples, and the grey area represent the $95 \%$ of the confidence region. 


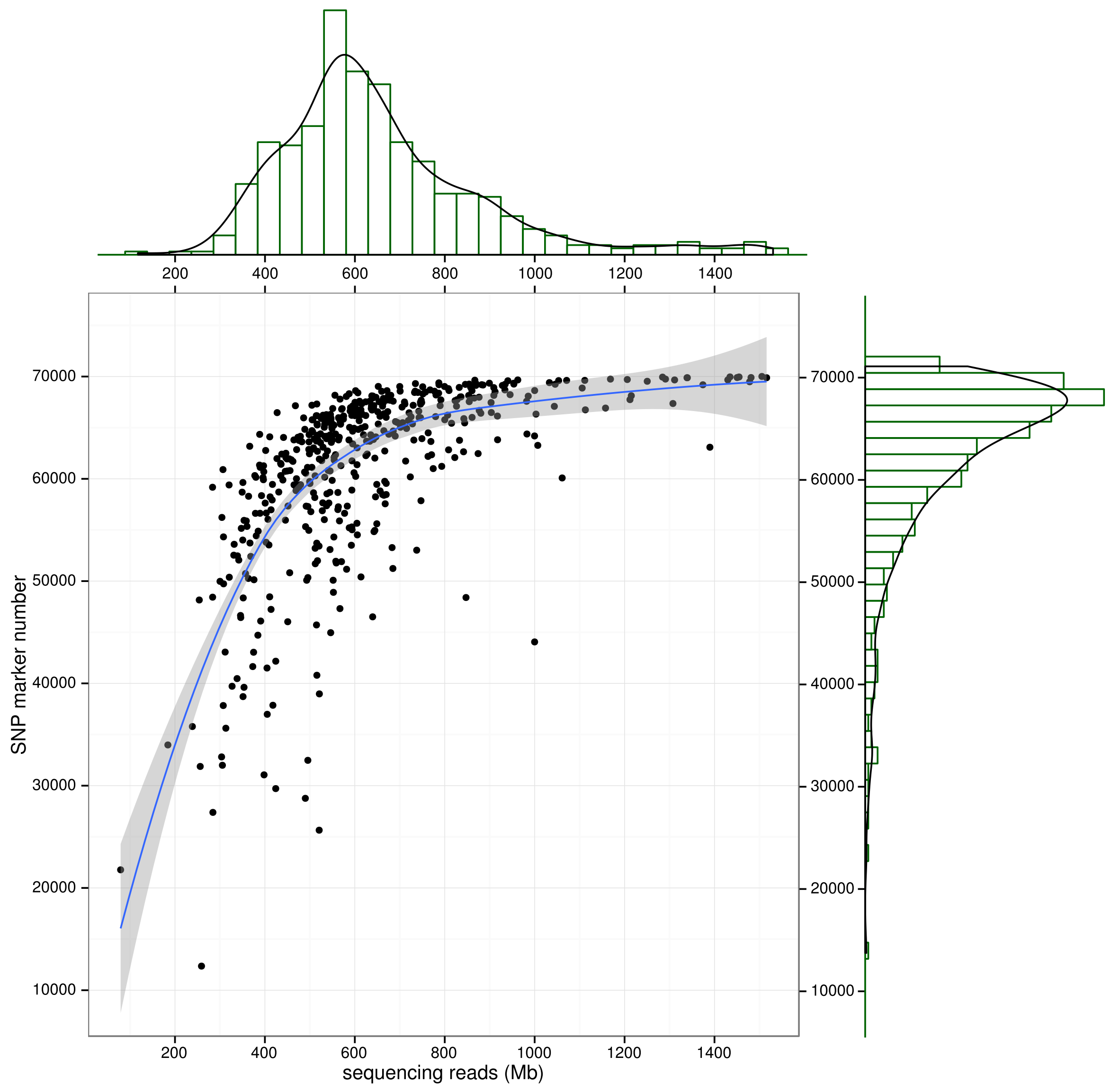


Figure 4 (on next page)

SNP distribution along chromosome.

SNP distribution along chromosome. The lines along the chromosomes represent SNP loci. The SNP location in exon, intron, UTR and intergenic regions are showed by red, blue, purple and green, respectively. 
PeerJ 


\section{Figure 5(on next page)}

Biological impact annotations of high quality SNP markers that shared by at least $80 \%$ of the population with 500 large yellow croakers.

Biological impact annotations of high quality SNP markers that shared by at least $80 \%$ of the population with 500 large yellow croakers. 


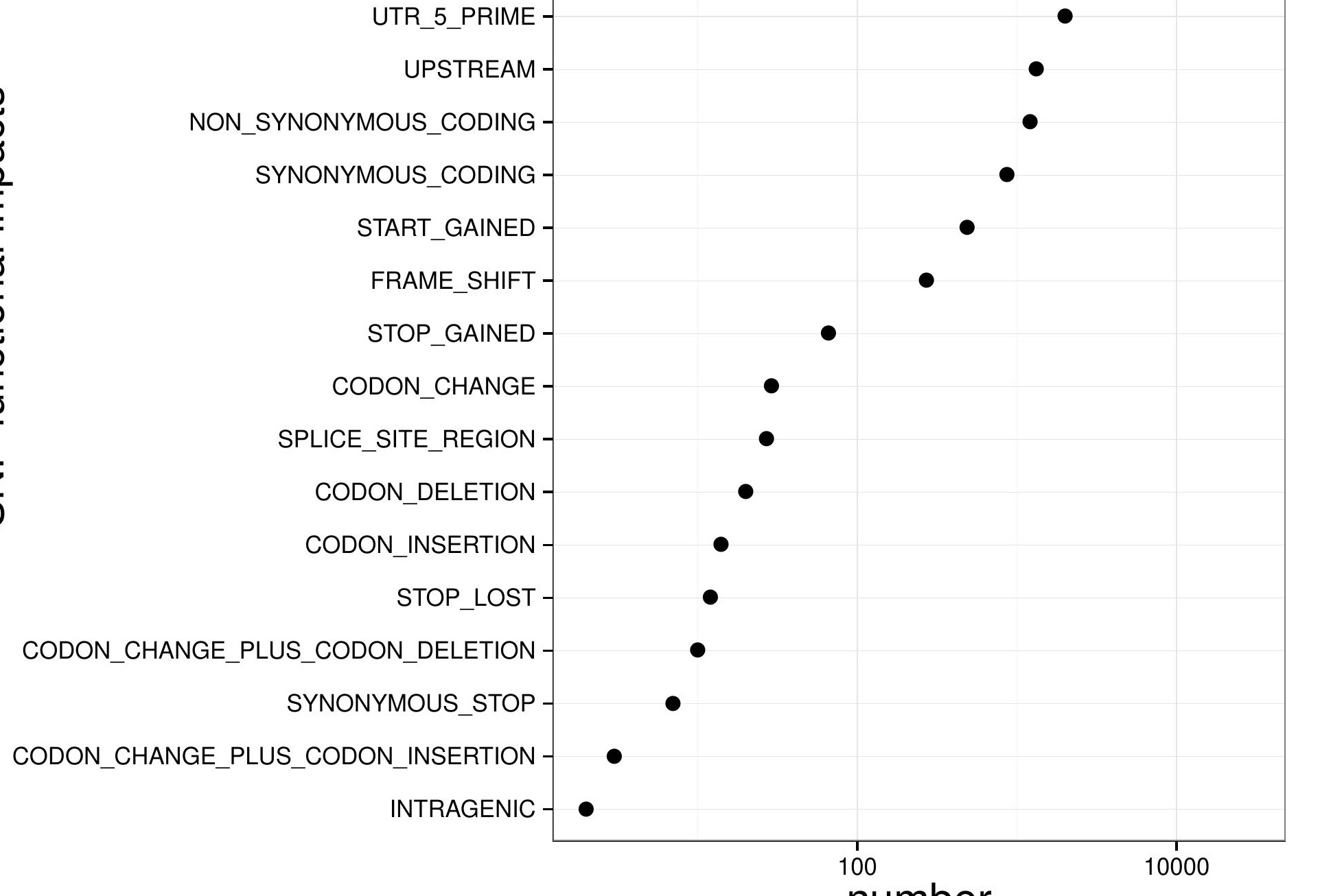




\section{Figure 6 (on next page)}

GWAS analysis on the muscle EPA and DHA content and the functional analysis therelated protein-coding genes.

\section{GWAS analysis on the muscle EPA and DHA content and the functional analysis}

therelated protein-coding genes. (A) The association results were illuminated in the Manhattan plot. The red line is the $p$-value threshold for significant markers; (B) KEGG pathway enrichment of functional genes; (C) GO term enrichment of related biological functions for the associated genes. 
(A)

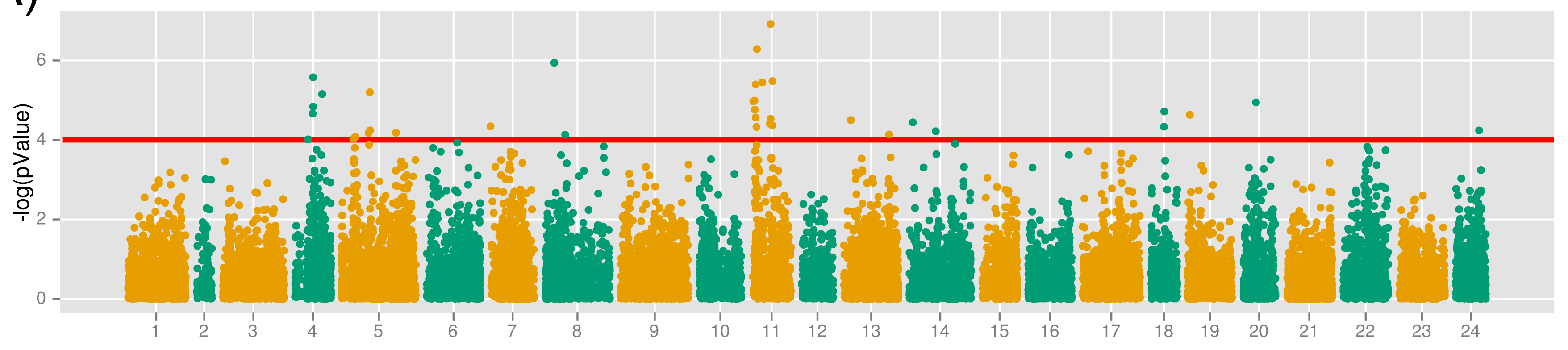

(B)

(C)

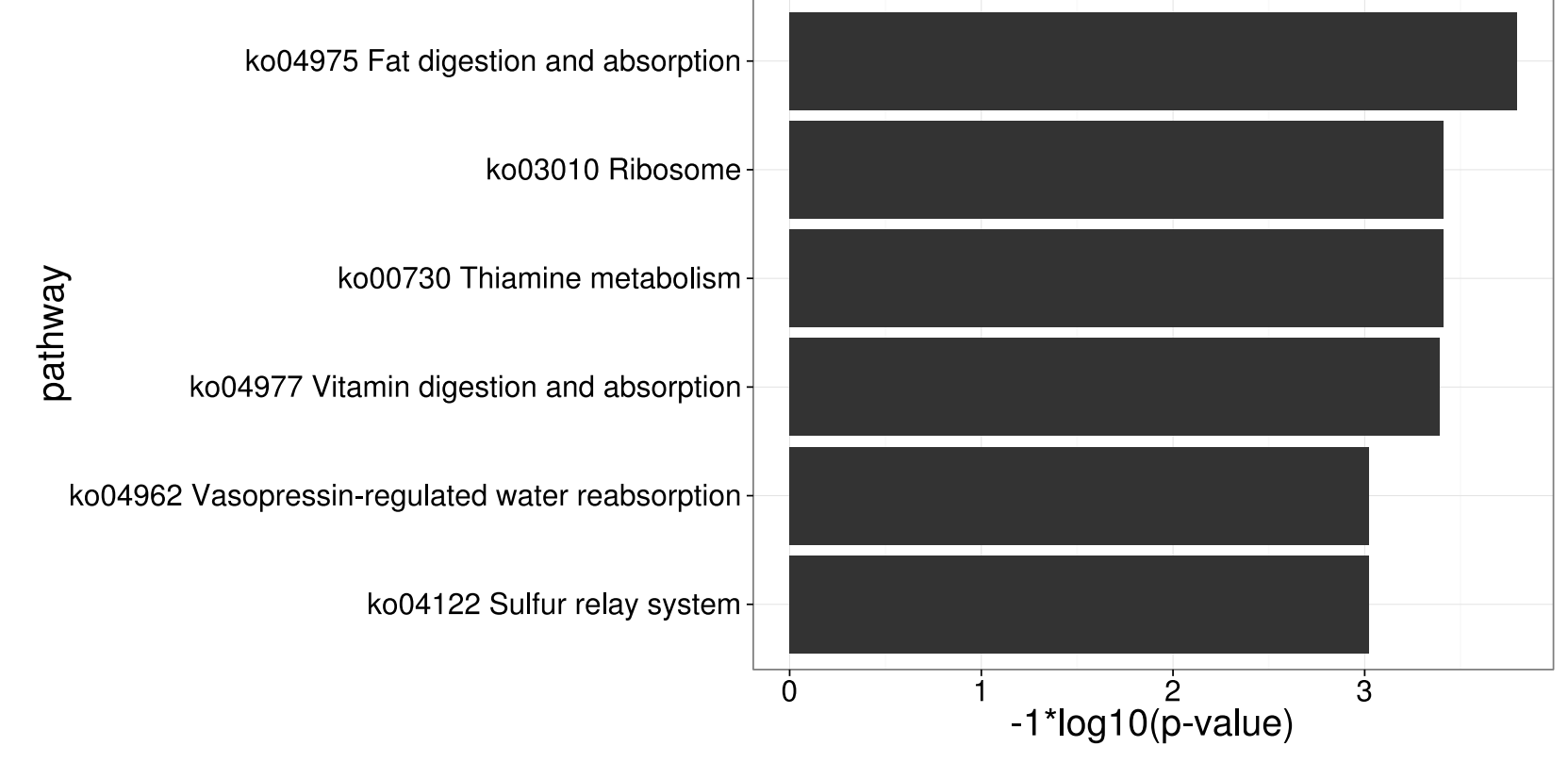

unsaturated fatty acid biosynthetic processunsaturated fatty acid metabolic process\&

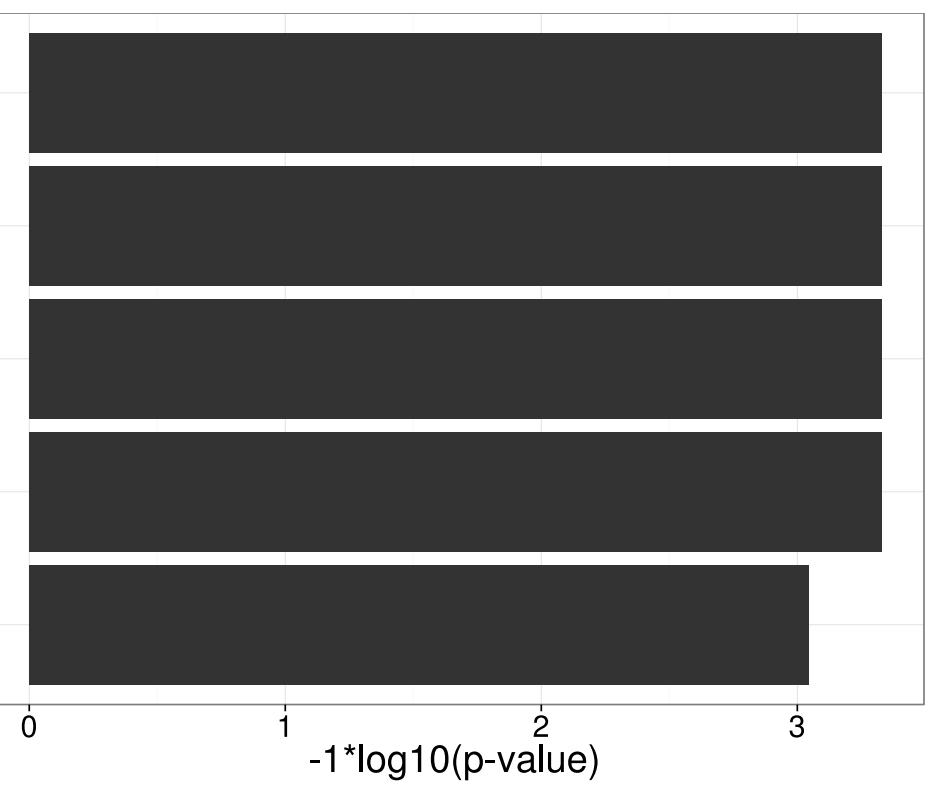

